\title{
A note on coeffective 1-differentiable cohomology
}

\author{
Cristian Ida and Sabin Mercheşan
}

Dedicated to Professor Mirela Ştefănescu at her 70-th anniversary

\begin{abstract}
After a brief review of some basic notions concerning 1-differentiable cohomology, named here $\widetilde{d}$-cohomology, we introduce a Lichnerowicz $\widetilde{d}-$ cohomology in a classical way. Next, following the classical study of coeffective cohomology, a special attention is paid to the study of some problems concerning coeffective cohomology in the graded algebra of 1differentiable forms. Also, the case of an almost contact metric $(2 n+1)-$ dimensional manifold is considered and studied in our context.
\end{abstract}

\section{Introduction}

The 1-differentiable cohomology was introduced and intensively studied by A. Lichnerowicz in $[16,9]$ in the context of symplectic and contact manifolds and in $[17,18]$ in the context of Poisson or Jacobi manifolds. Further signifiant developments of a such cohomology in the context of Lichnerowicz-Jacobi cohomology are given by M. de León, B. López, J. C. Marrero and E. Padrón, see for instance $[13,14,15]$. Here we consider the 1-differentiable cohomology of a manifold as follows: for every 1-form $\eta$ on a smooth manifold $M$ we define a coboundary operator $\widetilde{d}$ on the complex $\widetilde{\Omega}^{\bullet}(M)=\Omega^{\bullet}(M) \oplus \Omega^{\bullet-1}(M)$ by $\widetilde{d}(\varphi, \psi)=(d \varphi-d \eta \wedge \psi,-d \psi)$, where $\Omega^{\bullet}(M)=\oplus_{p \geq 0} \Omega^{p}(M) ; \Omega^{p}(M)$ is

Key Words: 1-differentiable form, coeffective cohomology, almost contact structure.

2010 Mathematics Subject Classification: Primary 58A10, 58A12.; Secondary 53D15.

Received: August 2013

Accepted:November 2013 
the space of $p$-forms on $M$. The resulting cohomology is named here $\widetilde{d}-$ cohomology of $M$. Also, we notice that an harmonic and $C$-harmonic theory of 1-differentiable forms on Sasakian manifolds with respect to the operator $\widetilde{d}$ is recently studied in [12].

The coeffective cohomology was introduced by T. Bouché [3] for symplectic manifolds. Further signifiant developments are given by D. Chinea, M. de León and J. C. Marrero for cosymplectic manifolds [6] and M. Fernández, R. Ibáñez, M. de León for contact manifolds [7] and other papers by these authors.

The purpose of this note is to extend the study of coeffective cohomology in the context of 1-differentiable forms.

The paper is organized as follows. In Section 2, after a briefly review of some basic notions concerning to 1-differentiable cohomology (or $\widetilde{d}$-cohomology associated to an one form $\eta$ ), a vanishing invariant $\widetilde{d}$-class of order $2 p+1, p=1, \ldots,\left[\frac{n}{2}\right]$ is defined in terms of the 1 -form $\eta$ and following the general study of Lichnerowicz cohomology (also known as Morse-Novikov cohomology) we define a Lichnerowicz $\widetilde{d}$-cohomology in the graded algebra of 1-differentiable forms $\widetilde{\Omega} \bullet(M)$. Also some vanishing Lichnerowicz $\widetilde{d}$-classes are given. In Section 3 are given the main results of the paper. Taking into account the classical construction of coeffective cohomologies which is strongly related to closed forms, $[3,6,7,8]$, we give some coeffective cohomologies in the graded algebra $\left(\widetilde{\Omega}^{\bullet}(M), \widetilde{\wedge}\right)$ and some relation with $\widetilde{d}$-cohomology. Since $(\eta, 1)$ is $\widetilde{d}-$ closed, firstly we define and we study an $(\eta, 1)$-coeffective cohomology. Next using the fact that $(d \eta, \eta)$ is closed with respect to Lichnerowicz $\widetilde{d}$-differential $\widetilde{d}_{(\eta, 1)}=\widetilde{d}+(\eta, 1) \widetilde{\wedge}$ we define and we study a $(d \eta, \eta)$-coeffective cohomology. Also, the case when $\eta$ is the fundamental 1-form of an almost contact metric $(2 n+1)$-dimensional manifold is considered and studied. We obtain that the $(d \eta, \eta)$-coeffective cohomology groups of an almost contact metric manifold of finite type have finite dimension (called the $(d \eta, \eta)$-coeffective numbers and denoted by $\left.\widetilde{c}_{p}(M,(d \eta, \eta))\right)$. Also, in this case, we prove that the $(d \eta, \eta)-$ coeffective numbers are bounded by topological numbers depending on the Betti numbers of the manifold. The methods used here are similarly and closely related to those used by $[6,7,8]$.

\section{1-differentiable $p$-forms and 1-differentiable cohomologies}

\section{$2.1 \quad \widetilde{d}$-cohomology}

Let us consider the field $\Omega^{0}(M)=\mathcal{F}(M)$ of smooth real valued functions defined on $M$. For each $p=1, \ldots, n=\operatorname{dim} M$ denote by $\Omega^{p}(M)$ the module of $p$-forms on $M$ and by $\Omega(M)=\oplus_{p \geq 0} \Omega^{p}(M)$ the exterior algebra of $M$. 
We denote $\widetilde{\Omega}^{p}(M)=\Omega^{p}(M) \oplus \Omega^{p-1}(M)$ and its elements are pair forms $(\varphi, \psi)$ called 1-differentiable $p$-forms (after a terminology used in [16]). As in formula (5.5) from [17], we can define a wedge product of 1-differentiable forms $\widetilde{\wedge}: \widetilde{\Omega}^{p}(M) \times \widetilde{\Omega}^{p^{\prime}}(M) \rightarrow \widetilde{\Omega}^{p+p^{\prime}}(M)$ by:

$$
(\varphi, \psi) \widetilde{\wedge}\left(\varphi^{\prime}, \psi^{\prime}\right)=\left(\varphi \wedge \varphi^{\prime},(-1)^{p} \varphi \wedge \psi^{\prime}+\psi \wedge \varphi^{\prime}\right)
$$

to be the exterior product on the space $\widetilde{\Omega} \bullet(M)$, where $(\varphi, \psi) \in \widetilde{\Omega}^{p}(M)$, $\left(\varphi^{\prime}, \psi^{\prime}\right) \in \widetilde{\Omega}^{p^{\prime}}(M)$. By this definition, we notice that for an 1-differentiable 0 -form $(f, 0)$, where $f$ is a smooth function on $M$ we have $(f, 0) \cdot(\varphi, \psi)=$ $(f \varphi, f \psi)$. Also, one easily verifies that:

$$
\begin{gathered}
(\varphi, \psi) \widetilde{\wedge}\left(\left(\varphi^{\prime}, \psi^{\prime}\right)+\left(\varphi^{\prime \prime}, \psi^{\prime \prime}\right)\right)=(\varphi, \psi) \widetilde{\wedge}\left(\varphi^{\prime}, \psi^{\prime}\right)+(\varphi, \psi) \widetilde{\wedge}\left(\varphi^{\prime \prime}, \psi^{\prime \prime}\right), \\
(\varphi, \psi) \widetilde{\wedge}\left(\left(\varphi^{\prime}, \psi^{\prime}\right) \widetilde{\wedge}\left(\varphi^{\prime \prime}, \psi^{\prime \prime}\right)\right)=\left((\varphi, \psi) \widetilde{\wedge}\left(\varphi^{\prime}, \psi^{\prime}\right)\right) \widetilde{\wedge}\left(\varphi^{\prime \prime}, \psi^{\prime \prime}\right)
\end{gathered}
$$

and

$$
(\varphi, \psi) \widetilde{\wedge}\left(\varphi^{\prime}, \psi^{\prime}\right)=(-1)^{p p^{\prime}}\left(\varphi^{\prime}, \psi^{\prime}\right) \widetilde{\wedge}(\varphi, \psi),
$$

which say that $(\widetilde{\Omega} \bullet(M), \widetilde{\Lambda})$ is a graded algebra.

For any 1 -form $\eta$ on $M$ we define the following operator in $(\widetilde{\Omega} \bullet(M), \widetilde{\wedge})$ :

$$
\widetilde{d}: \widetilde{\Omega}^{p}(M) \rightarrow \widetilde{\Omega}^{p+1}(M), \widetilde{d}(\varphi, \psi)=(d \varphi-L \psi,-d \psi),
$$

where $L: \Omega^{p}(M) \rightarrow \Omega^{p+2}(M)$ is given by $L \varphi=d \eta \wedge \varphi$.

An easy calculation shows that $\widetilde{d^{2}}=\widetilde{0}$, where $\widetilde{0}:=(0,0)$.

Denote by $\widetilde{H}^{\bullet}(M)$ the cohomology of the differential complex $(\widetilde{\Omega} \bullet(M), \widetilde{d})$ called 1-differentiable cohomology of $M$ (or $\widetilde{d}$-cohomology of $M$ ).

Remark 2.1. If we replace $d \eta$ by any 2-closed form, a such differential complex may be defined on every manifold $M$ endowed with a closed 2-form, for instance symplectic or Kähler manifolds.

We notice that this complex has local cohomology at both $p=0$ and $p=1$. Specifically, we have $\operatorname{ker}\left\{\widetilde{d}: \widetilde{\Omega}^{0}(M) \rightarrow \widetilde{\Omega}^{1}(M)\right\}=\{(f, 0) \mid f=$ const. $\}$ and the cohomology at $p=1$ is generated by $(\eta, 1)$.

Proposition 2.1. The $\widetilde{d}$-class $[(\eta, 1)]$ is nonzero in $\widetilde{H}^{1}(M)$.

Proof. If we suppose that $[(\eta, 1)]=\widetilde{0}$ then there exists an 1-differentiable zero form $(f, 0) \in \widetilde{\Omega}^{0}(M)=\Omega^{0}(M) \oplus\{0\}$ such that $(\eta, 1)=\widetilde{d}(f, 0)$ that is imposible. 
Let us consider now, the mappings $\alpha: \Omega^{p}(M) \rightarrow \widetilde{\Omega}^{p}(M), \alpha(\varphi)=(\varphi, 0)$ and $\beta: \widetilde{\Omega}^{p}(M) \rightarrow \Omega^{p-1}(M), \beta(\varphi, \psi)=\psi$ for all $\varphi \in \Omega^{p}(M)$ and $\psi \in \Omega^{p-1}(M)$, respectively. Then, we have the following result which relates $\widetilde{H}^{\bullet}(M)$ with the de Rham cohomology $H_{d R}^{\bullet}(M)$.

Proposition 2.2. Let $M$ be a n-dimensional smooth manifold. Then:

(i) The mappings $\alpha$ and $\beta$ induce an exact sequence of complexes

$$
0 \longrightarrow\left(\Omega^{\bullet}(M), d\right) \stackrel{\alpha}{\longrightarrow}\left(\widetilde{\Omega}^{\bullet}(M), \widetilde{d}\right) \stackrel{\beta}{\longrightarrow}\left(\Omega^{\bullet-1}(M),-d\right) \longrightarrow 0 .
$$

(ii) This exact sequence induces a long exact cohomology sequence

$$
\ldots \longrightarrow H_{d R}^{p}(M) \stackrel{\alpha^{*}}{\longrightarrow} \widetilde{H}^{p}(M) \stackrel{\beta^{*}}{\longrightarrow} H_{d R}^{p-1}(M) \stackrel{\delta_{p-1}^{*}}{\longrightarrow} H_{d R}^{p+1}(M) \longrightarrow \ldots,
$$

where the connecting homomorphism $\delta_{p-1}^{*}$ is defined by

$$
\delta_{p-1}^{*}[\psi]=[-L \psi]=0, \text { for any }[\psi] \in H_{d R}^{p-1}(M) .
$$

From above proposition, one gets

Corollary 2.1. Let $M$ be a n-dimensional smooth manifold. Then, for all $p$, we have

$$
\widetilde{H}^{p}(M) \cong H_{d R}^{p}(M) \oplus H_{d R}^{p-1}(M) .
$$

Consequently, $\operatorname{dim} \widetilde{H}^{p}(M)=b_{p}(M)+b_{p-1}(M)$, where $b_{p}(M)$ is the $p$ th Betti number of $M$. In particular, $\widetilde{b}(M):=\operatorname{dim} \widetilde{H}^{p}(M)$ is a topological invariant of $M$, for all $p$. Also, by applying the Poincaré duality for the de Rham cohomology $H_{d R}^{\bullet}(M)$ in (2.5) we obtain the following Poincaré duality for our cohomology:

$$
\widetilde{H}^{p}(M) \cong\left(\widetilde{H}_{c}^{2 n+2-p}(M)\right)^{*},
$$

where the index " $c$ " denotes the cohomology with compact support.

Also, it is easy to see that $\widetilde{d}\left(\eta \wedge(d \eta)^{p},(d \eta)^{p}\right)=(0,0), p=1, \ldots,\left[\frac{n}{2}\right]$ and so we have an invariant $\widetilde{d}$-class of order $2 p+1$ of the $n$-dimensional smooth manifold $M$

$$
\left[\left(\eta \wedge(d \eta)^{p},(d \eta)^{p}\right)\right] \in \widetilde{H}^{2 p+1}(M), p=1, \ldots,\left[\frac{n}{2}\right] .
$$

By direct calculus we have $\left(\eta \wedge(d \eta)^{p},(d \eta)^{p}\right)=\widetilde{d}\left((d \eta)^{p},-\eta \wedge(d \eta)^{p-1}\right)$ which say that the $\widetilde{d}$-class $\left[\left(\eta \wedge(d \eta)^{p},(d \eta)^{p}\right)\right]$ vanish. 


\subsection{Lichnerowicz $\widetilde{d}$-cohomology}

As well as we seen the 1-differentiable 1 -form $(\eta, 1)$ is $\widetilde{d}$-closed. Thus, as in the classical Lichnerowicz cohomology (also known as Morse-Novikov cohomology) we define the following operator in the graded algebra $(\widetilde{\Omega} \bullet(M), \widetilde{\wedge})$ :

$$
\widetilde{d}_{(\eta, 1)}: \widetilde{\Omega}^{p}(M) \rightarrow \widetilde{\Omega}^{p+1}(M), \widetilde{d}_{(\eta, 1)}=\widetilde{d}+(\eta, 1) \widetilde{\wedge} .
$$

By direct calculus we obtain $\widetilde{d}_{(\eta, 1)}^{2}=\widetilde{0}$, hence we get a differential complex $\left(\widetilde{\Omega}^{\bullet}(M), \widetilde{d}_{(\eta, 1)}\right)$ called the Lichnerowicz complex of 1-differentiable forms; its cohomology $\widetilde{H}_{(\eta, 1)}^{\bullet}(M)$ is called Lichnerowicz $\widetilde{d}$-cohomology of 1-differentiable forms on $M$. Note that $\tilde{d}_{(\eta, 1)}$ does not satisfy the Leibniz property, since for any $(\varphi, \psi) \in \widetilde{\Omega}^{p}(M)$ and $\left(\varphi^{\prime}, \psi^{\prime}\right) \in \widetilde{\Omega}^{p^{\prime}}(M)$ we have

$$
\widetilde{d}_{(\eta, 1)}\left((\varphi, \psi) \widetilde{\wedge}\left(\varphi^{\prime}, \psi^{\prime}\right)\right)=\widetilde{d}(\varphi, \psi) \widetilde{\wedge}\left(\varphi^{\prime}, \psi^{\prime}\right)+(-1)^{p}(\varphi, \psi) \widetilde{\wedge} \widetilde{d}_{(\eta, 1)}\left(\varphi^{\prime}, \psi^{\prime}\right) .
$$

Thus the Lichnerowicz $\widetilde{d}$-cohomology $\widetilde{H}_{(\eta, 1)}^{\bullet}(M)$ does not have a ring structure. The formula $(2.9)$ also implies that $\widetilde{H}_{(\eta, 1)}^{\bullet}(M)$ is a $\widetilde{H}^{\bullet}(M)$-module.

We have

Proposition 2.3. The Lichnerowicz $\widetilde{d}$-cohomology depends only on the $\widetilde{d}-$ class of $(\eta, 1)$. In fact, we have the isomorphism $\widetilde{H}_{(\eta, 1)+\widetilde{d}(f, 0)}^{p}(M) \approx \widetilde{H}_{(\eta, 1)}^{p}(M)$.

Proof. Since

$$
\widetilde{d}_{(\eta, 1)}\left(\left(e^{f}, 0\right) \cdot(\varphi, \psi)\right)=\left(e^{f}, 0\right) \widetilde{d}_{(\eta, 1)+\widetilde{d}(f, 0)}(\varphi, \psi)
$$

it results that the map $[(\varphi, \psi)] \mapsto\left[\left(e^{f}, 0\right) \cdot(\varphi, \psi)\right]$ is an isomorphism between $\widetilde{H}_{(\eta, 1)+\widetilde{d}(f, 0)}^{p}(M)$ and $\widetilde{H}_{(\eta, 1)}^{p}(M)$.

By straightforward caculus we obtain

Proposition 2.4. For any $(\varphi, \psi) \in \widetilde{\Omega}^{p}(M)$ and $\left(\varphi^{\prime}, \psi^{\prime}\right) \in \widetilde{\Omega}^{p^{\prime}}(M)$ we have

$$
\widetilde{d}\left((\varphi, \psi) \widetilde{\wedge}\left(\varphi^{\prime}, \psi^{\prime}\right)\right)=\widetilde{d}_{(\eta, 1)}(\varphi, \psi) \widetilde{\wedge}\left(\varphi^{\prime}, \psi^{\prime}\right)+(-1)^{p}(\varphi, \psi) \widetilde{\wedge} \widetilde{d}_{-(\eta, 1)}\left(\varphi^{\prime}, \psi^{\prime}\right) .
$$

Consequently

$$
\widetilde{d}_{(\eta, 1)}(\varphi, \psi) \widetilde{\wedge} \widetilde{d}_{-(\eta, 1)}\left(\varphi^{\prime}, \psi^{\prime}\right)=\widetilde{d}\left((\varphi, \psi) \widetilde{\wedge} \widetilde{d}_{-(\eta, 1)}\left(\varphi^{\prime}, \psi^{\prime}\right)\right) .
$$

Formula (2.10) yields the induced map

$$
\widetilde{H}_{(\eta, 1)}^{\bullet}(M) \times \widetilde{H}_{-(\eta, 1)}^{\bullet}(M) \rightarrow \widetilde{H}^{\bullet}(M) .
$$


Now, using (2.1), (2.8) and (2.9), a straightforward calculus leads to

$$
\widetilde{d}_{(\eta, 1)}\left((d \eta)^{p}, \eta \wedge(d \eta)^{p-1}\right)=\widetilde{0}, \widetilde{d}_{(\eta, 1)}\left(\eta \wedge(d \eta)^{p-1},(d \eta)^{p-1}\right)=\widetilde{0} .
$$

Thus, we can define two cohomology classes

$$
\left[\left((d \eta)^{p}, \eta \wedge(d \eta)^{p-1}\right)\right] \in \widetilde{H}_{(\eta, 1)}^{2 p}(M),\left[\left(\eta \wedge(d \eta)^{p-1},(d \eta)^{p-1}\right)\right] \in \widetilde{H}_{(\eta, 1)}^{2 p-1}(M)
$$

called the Lichnerowicz $\widetilde{d}$-classes of $M$.

Also, it is easy to see that

$$
\left((d \eta)^{p}, \eta \wedge(d \eta)^{p-1}\right)=\widetilde{d}_{(\eta, 1)}\left(\frac{\eta \wedge(d \eta)^{p-1}}{2},-\frac{(d \eta)^{p-1}}{2}\right)
$$

and

$$
\left(\eta \wedge(d \eta)^{p-1},(d \eta)^{p-1}\right)=\widetilde{d}_{(\eta, 1)}\left(\frac{(d \eta)^{p-1}}{2},-\frac{\eta \wedge(d \eta)^{p-2}}{2}\right)
$$

which say that the Lichnerowicz $\widetilde{d}$-classes from (2.13) vanishes.

The operator $\widetilde{d}_{(\eta, 1)}$ will be an important tool in the study of a $(d \eta, \eta)$ coeffective cohomology in the next section.

\section{Coeffective $\widetilde{d}$-cohomology}

Let us consider again $\eta$ a differential one form on $M$. Taking into account the classical construction of coeffective cohomologies which is strongly related to closed forms, see for instance $[7,8]$, the aim of this section is to give some coeffective cohomologies in the graded algebra $(\widetilde{\Omega} \bullet(M), \widetilde{\Lambda})$ and some relation with $\widetilde{d}$-cohomology. Since $(\eta, 1)$ is $\widetilde{d}$-closed, firstly we define and we study an $(\eta, 1)$-coeffective cohomology. Next using the fact that $(d \eta, \eta)$ is closed with respect to Lichnerowicz differential $\widetilde{d}_{(\eta, 1)}$ we define and we study a $(d \eta, \eta)$ coeffective cohomology. Also, the case when $\eta$ is a contact form of an almost contact metric $(2 n+1)$-dimensional manifold is considered and studied.

\section{$3.1(\eta, 1)$-coeffective $\widetilde{d}$-cohomology}

We define the operator

$$
\widetilde{L}_{(\eta, 1)}: \widetilde{\Omega}^{p}(M) \rightarrow \widetilde{\Omega}^{p+1}(M), \widetilde{L}_{(\eta, 1)}(\varphi, \psi)=(\varphi, \psi) \widetilde{\wedge}(\eta, 1) .
$$

The space

$$
\widetilde{\mathcal{A}}_{(\eta, 1)}^{p}(M)=\operatorname{ker}\left\{\widetilde{L}_{(\eta, 1)}: \widetilde{\Omega}^{p}(M) \rightarrow \widetilde{\Omega}^{p+1}(M)\right\}
$$


is called the subspace of $(\eta, 1)$-coeffective 1-differentiable forms on the smooth manifold $M$. Since $(\eta, 1)$ is $\widetilde{d}$-closed, $\widetilde{L}_{(\eta, 1)}$ and $\widetilde{d}$ commute, which implies that $\left(\widetilde{\mathcal{A}}_{(\eta, 1)}^{\bullet}(M), \widetilde{d}\right)$ is a differential subcomplex of the differential complex $\left(\widetilde{\Omega}^{\bullet}(M), \widetilde{d}\right)$. Its cohomology $\widetilde{H}^{p}\left(\widetilde{\mathcal{A}}_{(\eta, 1)}(M)\right)$ is called $(\eta, 1)$-coeffective $\widetilde{d}-$ cohomology of $M$. If this cohomology is finite, we define the $(\eta, 1)$-coeffective numbers by $\widetilde{c}_{p}(M,(\eta, 1))=\operatorname{dim} \widetilde{H}^{p}\left(\widetilde{\mathcal{A}}_{(\eta, 1)}(M)\right)$.

In the following we relate the $(\eta, 1)$-coeffective $\widetilde{d}$-cohomology with the $\widetilde{d}-$ cohomology by means of a long exact sequence in cohomology. Consider the following natural short exact sequence for any degree $p$ :

$$
\widetilde{0} \longrightarrow \operatorname{ker} \widetilde{L}_{(\eta, 1)}=\widetilde{\mathcal{A}}_{(\eta, 1)}^{p}(M) \stackrel{\widetilde{i}}{\longrightarrow} \widetilde{\Omega}^{p}(M) \stackrel{\widetilde{L}_{(\eta, 1)}}{\longrightarrow} \operatorname{Im}^{p+1} \widetilde{L}_{(\eta, 1)} \longrightarrow \widetilde{0} .
$$

Since $\widetilde{L}_{(\eta, 1)}$ and $\widetilde{d}$ commute, (3.2) becomes a short exact sequence of differential complexes.

Therefore, we can consider the associated long exact sequence in cohomology:

$$
\begin{gathered}
\ldots \longrightarrow \widetilde{H}^{p}\left(\widetilde{\mathcal{A}}_{(\eta, 1)}(M)\right) \stackrel{\widetilde{i}^{*}}{\longrightarrow} \widetilde{H}^{p}(M) \stackrel{\widetilde{L}_{(\eta, 1)}^{*}}{\longrightarrow} \\
\widetilde{H}^{p+1}\left(\operatorname{Im} \widetilde{L}_{(\eta, 1)}\right) \stackrel{\widetilde{\delta}_{p}^{*}}{\longrightarrow} \widetilde{H}^{p+1}\left(\widetilde{\mathcal{A}}_{(\eta, 1)}(M)\right) \longrightarrow \ldots
\end{gathered}
$$

where $\widetilde{i}^{*}$ and $\widetilde{L}_{(\eta, 1)}^{*}$ are the homomorphisms induced in cohomology by $\widetilde{i}$ and $\widetilde{L}_{(\eta, 1)}$, respectively, and $\widetilde{\delta}_{p}^{*}$ is the connecting homomorphism defined by

$$
\widetilde{\delta}_{p}^{*}[(\varphi, \psi)]=\left[\widetilde{d}\left(\varphi^{\prime}, \psi^{\prime}\right)\right]
$$

for any $\left(\varphi^{\prime}, \psi^{\prime}\right) \in \widetilde{\Omega}^{p}(M)$ such that $\widetilde{L}_{(\eta, 1)}\left(\varphi^{\prime}, \psi^{\prime}\right)=(\varphi, \psi)$.

If $\eta$ is an 1 -form without zeros we have

$$
\widetilde{H}^{0}\left(\widetilde{\mathcal{A}}_{(\eta, 1)}(M)\right) \cong\{\widetilde{0}\} .
$$

Moreover, since $(\varphi, \psi) \widetilde{\wedge}(\eta, 1)=(0,0)$ implies $(\varphi, \psi)=(\omega, \theta) \widetilde{\wedge}(\eta, 1)$ we deduce that

$$
\operatorname{ker}\left\{\widetilde{L}_{(\eta, 1)}: \widetilde{\Omega}^{p}(M) \rightarrow \widetilde{\Omega}^{p+1}(M)\right\}=\operatorname{Im}\left\{\widetilde{L}_{(\eta, 1)}: \widetilde{\Omega}^{p-1}(M) \rightarrow \widetilde{\Omega}^{p}(M)\right\} .
$$

Now decompose the long exact sequence (3.3) in the following short exact sequences:

$$
\widetilde{0} \longrightarrow \operatorname{Im} \widetilde{i}^{*}=\operatorname{ker} \widetilde{L}_{(\eta, 1)}^{*} \stackrel{i}{\longrightarrow} \widetilde{H}^{p}(M) \stackrel{\widetilde{L}_{(\eta, 1)}^{*}}{\longrightarrow} \operatorname{Im} \widetilde{L}_{(\eta, 1)}^{*} \longrightarrow \widetilde{0} .
$$


Then we deduce the formula:

$$
\widetilde{b}_{p}(M)=\operatorname{dim}\left(\operatorname{ker} \widetilde{L}_{(\eta, 1)}^{*}\right)+\operatorname{dim}\left(\operatorname{Im} \widetilde{L}_{(\eta, 1)}^{*}\right) .
$$

From (3.5) we obtain the following result:

Proposition 3.1. Let $M$ be a n-dimensional smooth manifold. Asume that $\eta$ is an 1-form without zeros on $M$ and $(\eta, 1)$-coeffective $\widetilde{d}$-cohomology is finite. Then we have

$$
\widetilde{b}_{p}(M) \leq \widetilde{c}_{p}(M,(\eta, 1))+\widetilde{c}_{p+1}(M,(\eta, 1))
$$

for all $p$.

\section{$3.2(d \eta, \eta)$-coeffective $\widetilde{d}$-cohomology}

As in the previous subsection, the main purpose of this subsection is to construct a $(d \eta, \eta)$-coeffective $\widetilde{d}$-cohomology. Althouhg $(d \eta, \eta)$ is not $\widetilde{d}$-closed it is $\widetilde{d}_{(\eta, 1)}$-closed and this fact allow to construct an associated coeffective $\widetilde{d}_{-}$ cohomology. The case when $M$ is an almost contact manifold is also considered and studied in the next subsection.

Let us define the operator

$$
\widetilde{L}_{(d \eta, \eta)}: \widetilde{\Omega}^{p}(M) \rightarrow \widetilde{\Omega}^{p+2}(M), \widetilde{L}_{(d \eta, \eta)}(\varphi, \psi)=(\varphi, \psi) \widetilde{\wedge}(d \eta, \eta) .
$$

The space

$$
\widetilde{\mathcal{A}}_{(d \eta, \eta)}^{p}(M)=\operatorname{ker}\left\{\widetilde{L}_{(d \eta, \eta)}: \widetilde{\Omega}^{p}(M) \rightarrow \widetilde{\Omega}^{p+2}(M)\right\}
$$

is called the subspace of $(d \eta, \eta)$-coeffective 1-differentiable forms on $M$.

Taking into acount that $\widetilde{d}_{(\eta, 1)}(d \eta, \eta)=\widetilde{0}$ the relation $(2.9)$ say that

$$
\widetilde{d}_{(\eta, 1)}((\varphi, \psi) \widetilde{\wedge}(d \eta, \eta))=\widetilde{d}(\varphi, \psi) \widetilde{\wedge}(d \eta, \eta)
$$

or, equivalently

$$
\widetilde{d}_{(\eta, 1)} \widetilde{L}_{(d \eta, \eta)}=\widetilde{L}_{(d \eta, \eta)} \widetilde{d}
$$

The identity (3.8) suggests us to consider a family of operators $\widetilde{d}_{(k \eta, k)}, k \in \mathbb{R}$, which we abbreviate as $\widetilde{d}_{k}$ if no misunderstanding occurs. We get immediatly from (3.8)

$$
\widetilde{d}_{k} \widetilde{L}_{(d \eta, \eta)}^{p}=\widetilde{L}_{(d \eta, \eta)}^{p} \widetilde{d}_{k-p}, \forall p \geq 0,
$$

where $\widetilde{L}_{(d \eta, \eta)}^{0}=\left.\mathrm{Id}\right|_{\widetilde{\Omega}(M)}$. 
Now, the relation $(3.8)$ say that if $(\varphi, \psi) \in \widetilde{\mathcal{A}}_{(d \eta, \eta)}^{p}(M)$ then $\widetilde{d}(\varphi, \psi) \in$ $\widetilde{\mathcal{A}}_{(d \eta, \eta)}^{p+1}(M)$, hence $\left(\widetilde{\mathcal{A}}_{(d \eta, \eta)}^{p}(M), \widetilde{d}\right)$ is a differential subcomplex of the differential complex $\left(\widetilde{\Omega}^{p}(M), \widetilde{d}\right)$. Its cohomology $\widetilde{H}^{p}\left(\widetilde{\mathcal{A}}_{(d \eta, \eta)}(M)\right)$ is called $(d \eta, \eta)$ coeffective $\widetilde{d}$-cohomology of $M$. If this cohomology is finite, then we define the $(d \eta, \eta)$-coeffective numbers by $\widetilde{c}_{p}(M,(d \eta, \eta))=\operatorname{dim} \widetilde{H}^{p}\left(\widetilde{\mathcal{A}}_{(d \eta, \eta)}(M)\right)$.

As in the case of $(\eta, 1)$-coeffective $\widetilde{d}$-cohomology, in the sequel we relate the $(d \eta, \eta)$-coeffective $\widetilde{d}$-cohomology with the $\widetilde{d}$-cohomology by means of a long exact sequence in cohomology. Consider the following natural short exact sequence for any degree $p$ :

$$
\widetilde{0} \longrightarrow \operatorname{ker} \widetilde{L}_{(d \eta, \eta)}=\widetilde{\mathcal{A}}_{(d \eta, \eta)}^{p}(M) \stackrel{\widetilde{i}}{\longrightarrow} \widetilde{\Omega}^{p}(M) \stackrel{\widetilde{L}_{(d \eta, \eta)}}{\longrightarrow} \operatorname{Im}^{p+2} \widetilde{L}_{(d \eta, \eta)} \longrightarrow \widetilde{0} .
$$

By means of $(3.9)$, for $k=0$ and $p=1$, we obtain $\widetilde{d L}_{(d \eta, \eta)}=\widetilde{L}_{(d \eta, \eta)} \widetilde{d}_{-(\eta, 1)}$ which say that if $(\varphi, \psi) \in \operatorname{Im}^{p} \widetilde{L}_{(d \eta, \eta)}$ then $\widetilde{d}(\varphi, \psi) \in \operatorname{Im}^{p+1} \widetilde{L}_{(d \eta, \eta)}$, hence $\left(\operatorname{Im}^{p} \widetilde{L}_{(d \eta, \eta)}, \widetilde{d}\right)$ is a subcomplex of the differential complex $\left(\widetilde{\Omega}^{p}(M), \widetilde{d}\right)$. Thus, (3.10) becomes a short exact sequence of differential complexes.

Therefore, we can consider the associated long exact sequence in cohomology:

$$
\begin{gathered}
\ldots \longrightarrow \widetilde{H}^{p}\left(\widetilde{\mathcal{A}}_{(d \eta, \eta)}(M)\right) \stackrel{\widetilde{i}^{*}}{\longrightarrow} \widetilde{H}^{p}(M) \stackrel{\widetilde{L}_{(d \eta, \eta)}^{*}}{\longrightarrow} \\
\widetilde{H}^{p+2}\left(\operatorname{Im} \widetilde{L}_{(d \eta, \eta)}\right) \stackrel{\widetilde{\Delta}_{p+2}^{*}}{\longrightarrow} \widetilde{H}^{p+1}\left(\widetilde{\mathcal{A}}_{(d \eta, \eta)}(M)\right) \longrightarrow \ldots
\end{gathered}
$$

where $\widetilde{i}^{*}$ and $\widetilde{L}_{(d \eta, \eta)}^{*}$ are the homomorphisms induced in cohomology by $\widetilde{i}$ and $\widetilde{L}_{(d \eta, \eta)}$, respectively, and $\widetilde{\Delta}_{p+2}^{*}$ is the connecting homomorphism.

\subsection{The almost contact case}

In this subsection we consider that $\eta$ is the almost contact 1 -form of a $(2 n+1)$ dimensional almost contact manifold $M$.

Let us recall the following fundamental result due to [5]:

Proposition 3.2. Let $(M, F, \xi, \eta, g)$ be a $(2 n+1)$-dimensional almost contact metric manifold, (for necessary definitions see for instance [1, 4, 20]). Then the map

$$
L: \Omega^{p}(M) \rightarrow \Omega^{p+2}(M), L \varphi=\varphi \wedge d \eta
$$

is injective for $p \leq n-1$, and surjective for $p \geq n$.

Using the above proposition, we have 
Proposition 3.3. Let $(M, F, \xi, \eta, g)$ be a $(2 n+1)$-dimensional almost contact metric manifold. Then the map $\widetilde{L}_{(d \eta, \eta)}$ given in (3.7) is injective for $p \leq n-1$, and surjective for $p \geq n+1$.

Proof. Using (2.1) we have $\widetilde{L}_{(d \eta, \eta)}(\varphi, \psi)=(L \varphi, \eta \wedge \varphi+L \psi)$. Now, from $\widetilde{L}_{(d \eta, \eta)}\left(\varphi_{1}, \psi_{1}\right)=\widetilde{L}_{(d \eta, \eta)}\left(\varphi_{2}, \psi_{2}\right)$ we obtain

$$
L \varphi_{1}=L \varphi_{2}, \eta \wedge \varphi_{1}+L \psi_{1}=\eta \wedge \varphi_{2}+L \psi_{2}
$$

and by Proposition 3.2 if $p \leq n-1, L$ is injective and from the first relation of (3.12) it results that $\varphi_{1}=\varphi_{2}$. Replacing in the second relation of (3.12) we obtain $\psi_{1}=\psi_{2}$, and so $\left(\varphi_{1}, \psi_{1}\right)=\left(\varphi_{2}, \psi_{2}\right)$ which say that $\widetilde{L}_{(d \eta, \eta)}$ is injective for $p \leq n-1$.

Taking into account that for any $p \geq n, L$ is surjective we obtain that for any $\varphi^{\prime} \in \Omega^{p+2}(M)$ there is $\varphi \in \Omega^{p}(M)$ such that $\varphi^{\prime}=L \varphi$. Also, for $\varphi$ as above, if $p-1 \geq n$ then for any $\psi^{\prime} \in \Omega^{p+1}(M)$ there is $\psi \in \Omega^{p-1}$ such that $\psi^{\prime}-\eta \wedge \varphi=L \psi$, and so we conclude that if $p \geq n+1$ then for any $\left(\varphi^{\prime}, \psi^{\prime}\right) \in \widetilde{\Omega}^{p+2}(M)$ there exists $(\varphi, \psi) \in \widetilde{\Omega}^{p}(M)$ such that $\widetilde{L}_{(d \eta, \eta)}(\varphi, \psi)=$ $\left(\varphi^{\prime}, \psi^{\prime}\right)$, which say that $\widetilde{L}_{(d \eta, \eta)}$ is surjective for $p \geq n+1$.

Corollary 3.1. $\widetilde{\mathcal{A}}_{(d \eta, \eta)}^{p}(M)=\{\widetilde{0}\}$, for $p \leq n-1$, and as a consequence

$$
\widetilde{H}^{p}\left(\widetilde{\mathcal{A}}_{(d \eta, \eta)}(M)\right)=\{0\}, \forall p=0,1, \ldots, n-1,
$$

or equivalently $\widetilde{c}_{p}(M,(d \eta, \eta))=0$, for any $p=0,1, \ldots, n-1$.

By Proposition 3.3 we have that $\operatorname{Im}^{p+2} \widetilde{L}_{(d \eta, \eta)}=\widetilde{\Omega}^{p+2}(M)$, for $p \geq n+1$. As a consequence, we have

$$
\widetilde{H}^{p+2}\left(\operatorname{Im} \widetilde{L}_{(d \eta, \eta)}\right)=\widetilde{H}^{p+2}(M), \forall p \geq n+2 .
$$

Furthermore, for $p \geq n+2$, the long exact sequence in cohomology (3.11) may be expressed as

$$
\begin{aligned}
& \ldots \longrightarrow \widetilde{H}^{p}\left(\widetilde{\mathcal{A}}_{(d \eta, \eta)}(M)\right) \stackrel{\widetilde{i}^{*}}{\longrightarrow} \widetilde{H}^{p}(M) \stackrel{\widetilde{L}_{(d \eta, \eta)}^{*}}{\longrightarrow} \\
& \widetilde{H}^{p+2}(M) \stackrel{\widetilde{\Delta}_{p+2}^{*}}{\longrightarrow} \widetilde{H}^{p+1}\left(\widetilde{\mathcal{A}}_{(d \eta, \eta)}(M)\right) \longrightarrow \ldots
\end{aligned}
$$

Now, we shall decompose the long exact sequence (3.13) in 5-terms exact sequences:

$$
0 \rightarrow \operatorname{Im} \widetilde{\Delta}_{p+1}^{*} \stackrel{i}{\longrightarrow} \widetilde{H}^{p}\left(\widetilde{\mathcal{A}}_{(d \eta, \eta)}(M)\right) \stackrel{\widetilde{i}^{*}}{\longrightarrow} \widetilde{H}^{p}(M) \stackrel{\widetilde{L}_{(d \eta, \eta)}^{*}}{\longrightarrow}
$$




$$
\widetilde{H}^{p+2}(M) \stackrel{\widetilde{\Delta}_{p+2}^{*}}{\longrightarrow} \operatorname{Im} \widetilde{\Delta}_{p+2}^{*} \longrightarrow 0
$$

where $\operatorname{Im} \widetilde{\Delta}_{p+1}^{*}=\operatorname{ker} \widetilde{i}^{*}$.

If $M$ is of finite type, the de Rham cohomology groups have finite dimension, and so $\widetilde{b}_{p}(M)=b_{p}(M)+b_{p-1}(M)$ is finite. Since $0 \leq \operatorname{dim}\left(\operatorname{Im} \widetilde{\Delta}_{p}^{*}\right) \leq$ $\widetilde{b}_{p}(M)$, for $p \geq n+4$ we have the following result:

Proposition 3.4. Let $(M, F, \xi, \eta, g)$ be a $(2 n+1)$-dimensional almost contact metric manifold of finite type, then the $(d \eta, \eta)$-coeffective $\widetilde{d}$-cohomology group $\widetilde{H}^{p}\left(\widetilde{\mathcal{A}}_{(d \eta, \eta)}(M)\right)$ has finite dimension, for $p \geq n+3$.

From (3.14), we have

$\widetilde{b}_{p+2}(M)-\widetilde{b}_{p}(M)=\operatorname{dim}\left(\operatorname{Im} \widetilde{\Delta}_{p+1}^{*}\right)-\operatorname{dim} \widetilde{H}^{p}\left(\widetilde{\mathcal{A}}_{(d \eta, \eta)}(M)\right)+\operatorname{dim}\left(\operatorname{Im} \widetilde{\Delta}_{p+2}^{*}\right)$, for $p \geq n+3$, from which we deduce

$$
\widetilde{c}_{p}(M,(d \eta, \eta))=\operatorname{dim}\left(\operatorname{Im} \widetilde{\Delta}_{p+1}^{*}\right)+\widetilde{b}_{p}(M)-\widetilde{b}_{p+2}(M)+\operatorname{dim}\left(\operatorname{Im} \widetilde{\Delta}_{p+2}^{*}\right) .
$$

Now, as a consequence of (3.15), we obtain

Theorem 3.1. For $p \geq n+3$, we have

$$
\widetilde{b}_{p}(M)-\widetilde{b}_{p+2}(M) \leq \widetilde{c}_{p}(M,(d \eta, \eta)) \leq \widetilde{b}_{p}(M)+\widetilde{b}_{p+1}(M) .
$$

Now, using Proposition 3.1 we obtain

Corollary 3.2. Let $(M, F, \xi, \eta, g)$ be a $(2 n+1)$-dimensional almost contact metric manifold of finite type, then

$$
\widetilde{c}_{p}(M,(d \eta, \eta)) \leq \widetilde{c}_{p}(M,(\eta, 1))+2 \widetilde{c}_{p+1}(M,(\eta, 1))+\widetilde{c}_{p+2}(M,(\eta, 1))
$$

for every $p \geq n+3$.

\section{Acknowledgments}

The authors wishes to express their deep gratitude to the Organizing Committee of The International Conference $A$ new approach in theoretical and applied methods in algebra and analysis, Constanta, 4-6 April 2013, CNCS-UEFISCDI grant PN-II-ID-WE-2012-4-169. The first author is supported by the Sectorial Operational Program Human Resources Development (SOP HRD), financed from the European Social Fund and by the Romanian Government under the Project number POSDRU/ID134378 . 


\section{References}

[1] D. E. Blair, Riemannian geometry of contact and symplectic manifolds. Progress in Mathematics, 203, Birkhäuser Boston, Inc., Boston, MA, 2002.

[2] R. Bott, L. W. Tu, Differential Forms in Algebraic Topology. Graduate Text in Math., 82, Springer-Verlag, Berlin, 1982.

[3] T. Bouché, La cohomologie coeffective d'une variété symplectique. Bull. Sc. Math. 114 (1990) (2) 115-122.

[4] C. P. Boyer, K. Galicki, Sasakian geometry. Oxford Mathematical Monographs. Oxford University Press, Oxford, 2008.

[5] D. Chinea, M. de León, J. C. Marrero, Topology of cosymplectic manifolds. J. Math. Pures et Appl. 72 (1993) (6), 567-591.

[6] D. Chinea, M. de León, J. C. Marrero, Coeffective cohomology on cosymplectic manifolds. Bull. Sci. Math. 119 (1995)(1) 3-20.

[7] M. Fernández, R. Ibáñez, M. de León, Coeffective and de Rham cohomologies on almost contact manifolds. Diff. Geom. Appl. 8 (1998), 285-303.

[8] M. Fernández, R. Ibáñez, M. de León, The geometry of a closed form. Homotopy and Geometry, Banach Center Publ. Vol. 45, Inst. of Math. Polish Acad. Sci., Warszawa 1998, 155-167.

[9] M. Flato, A. Lichnerowicz, D. Sternheimer, Déformations 1différentiables des algèbres de Lie attachées à une variété symplectique ou de contact. Compositio Math., 31 no. 1 (1975), 47-82.

[10] C. Ida, M. Crasmareanu, A cohomology associated to a vector field. Submitted.

[11] C. Ida, S. Mercheşan, Some remarks about 1-differentiable cohomology of Sasakian manifolds. Proceedings of 12-th Conference on Applied Mathematics APLIMAT, 5-7 February 2013, Bratislava, ISBN 978-80-227-38651, Paper 33. To appear also in Aplimat-Journal of Applied Mathematics, 2013.

[12] C. Ida, S. Mercheşan, On harmonic and C-harmonic 1-differentiable forms on Sasakian manifolds. Mediterr. J. Math. 2014, DOI 10.1007/s00009-013-0314-9. In press. 
[13] M. de León, J.C. Marrero, E. Padrón, Lichnerowicz-Jacobi cohomology. J. Phys. A: Math. Gen., 30 (1997), 6029-6055.

[14] M. de León, B. López, J. C. Marrero, E. Padrón, Lichnerowicz-Jacobi cohomology and homology of Jacobi manifolds : modular class and duality. available to arXiv:math/9910079v1 [math.DG] 1999.

[15] M. de León, B. López, J. C. Marrero, E. Padrón, On the computation of the Lichnerowicz-Jacobi cohomology, J. Geom. Phys. 44 (2003), 507-522.

[16] A. Lichnerowicz, Cohomologie 1-differentiable des algébres de Lie attachées a une variété symplectique ou de contact. J. Math. pures et appl., 53 (1974), 459-484.

[17] A. Lichnerowicz, Les variétés de Poisson et leurs algébres de Lie associées. J. Diff. Geom. 12 (1977), 253-300.

[18] A. Lichnerowicz, Les variétés de Jacobi et leurs algébres de Lie associées. J. Math. pures et appl., 57 (1978), 453-488.

[19] S. P. Novikov, The Hamiltonian formalism and a multivalued analogue of Morse theory, (Russian), Uspekhi Mat. Nauk 37 (1982), 3-49.

[20] G. Pitiş, Contact Geometry: Sasaki manifolds, Kenmotsu manifolds. Manuscript (unpublished).

[21] I. Vaisman, Cohomology and differential forms. M. Dekker Publ. House, New York, 1973.

Cristian IDA,

Department of Mathematics and Computer Science,

Transilvania University of Braşov,

Braşov 500091, Str. Iuliu Maniu 50, România

Email: cristian.ida@unitbv.ro

Sabin MERCHEŞAN,

Department of Mathematics and Computer Science,

Transilvania University of Braşov,

Braşov 500091, Str. Iuliu Maniu 50, România

Email: s.merchesan@unitbv.ro 